\title{
HIGH SPEED NON-LINEAR CIRCUIT SIMULATION OF BIPOLAR JUNCTION TRANSISTORS
}

\author{
M.N. DOJA ${ }^{\mathrm{a}, *}$, MOINUDDIN $^{\mathrm{b}}$ and UMESH KUMAR ${ }^{\mathrm{b}}$ \\ ${ }^{a}$ Department of Electrical Engineering, Jamiamillia Islamia, India; \\ ${ }^{\mathrm{b}}$ IIT Delhi, India
}

(Received 30 October 1998; In final form 14 December 1998)

\begin{abstract}
This paper presents HIBTRA (High Speed Bipolar Transistor Analysis), a high speed non-linear bipolar transistor circuit simulation package. The paper discusses about the modelling of Bipolar Junction Transistor operated at high speed in the sinusoidal small signal and the transient region of operations. The package uses a high frequency model non-linear circuit elements for accurate analysis. The package also uses transistor's lumped circuit model to calculate devices electrical parameters, and it also does dynamic simulation. It also includes the conventional model as a special case. Model verification has also been done by simulation.
\end{abstract}

Keywords: HIBTRA; simulation; BPJT

\section{INTRODUCTION}

Bipolar transistors are used today in a variety of applications from power electronics and fast digital circuits to microwave amplifiers, both in integrated and discrete form. Considering the increasing level of integration to lower the costs, it is essential to use efficient CAD tools to achieve good design productivity and to predict the performance of the circuit under consideration.

For reliable circuit simulation, accurate model of devices to be used are essential. The modeling of bipolar transistors has been the subject

*Address for correspondence: C46, Eastend Apartments, Mayur Vihar Phase1 Ext., Delhi 110096, India. Tel.: 2717428, Fax: 6311261, e-mail: doja_el@jmi.ernet.in/ndoja@ hotmail.com 
of extensive study during the last few decades. Ebers and Moll [1] developed a model to describe the essential features of transistor operation. The charge control concept [2] was later used to formulate the Gummel-Poon model [3] which is the basis of most of the non-linear bipolar transistor models today.

Inspite of these advances it is difficult to combine non-linear and high frequency effects into a single model. This would be essentially important with regard to a microwave power transistor in class $\mathrm{C}$ operation near the transition frequency. One possibility is to use the Gummel-Poon model with external parasitic elements to increase its accuracy at high frequencies [4]. There are, however, reasons to believe that this approach is not entirely satisfactory.

It is required to use frequency-dependent elements values in the circuit models of microwave transistors if the models have to cover a wide range of frequencies. On the other hand, the small signal diffusion conductance and capacitance of a $p-n$ junction are also frequency dependent [5]. It is clear that this phenomenon must also appear in the bipolar transistor.

The use of higher order and dynamic circuit elements [6] opens up new possibilities in accurate non linear modeling. Philippow and Jakubenko [7] ) have presented a bipolar transistor model using higher order elements. Chua and Chang [8] have demonstrated the applicability of these elements to the high speed non linear modeling of $p$ - $n$ junction diodes. Sipila et al. [9] have extended the analysis to bipolar transistor in which an improved description of the base dynamics is presented.

The high speed bipolar transistor modeling via non-linear circuit elements is a more accurate modelling approach. The current existing CAD packages are not based on this model [9] HIBTRA incorporates the latest high frequency model of bipolar transistor presented by Sipila et al. [9] it also includes an analytical model, a physical model and other dynamic models.

The purpose of this paper is to present a software, which can be used for device simulation and differs from other softwares. We have studied the latest BJT models. HIBTRA inputs the physical parameters of the device and it also does dynamic analysis at the desired operating frequency. The user can be get the print out of the terminal current characteristics and other parameter values at the required speed. It also overcomes the difficulties involved in the transient measurement of the 
fast devices. The high frequency accurate model used take third order derivative of the input signal and it computes the recombination and charge current in the base region. The model has provision for sinusoidal and transient analysis of the equivalent circuit. It also uses a lumped bipolar transistor model in which starting from the diffusion equation in the base region, an electrical equivalent circuit model is realized. It also uses a Static Model which is based on the steady state analysis of transistor behavior. It also calculates various other transistor parameters like cut-off frequency, forward and reverse current gains, reverse saturation current, resistance and diffusion capacitances of the emitter and collector regions.

\section{MODEL DISCUSSION}

HIBTRA uses four models, 1) Analytical Model based on improved description of base dynamics, 2) Numerical model, 3) Lumped Transistor model and 4) Static Transistor Model.

\subsection{Analytical Model}

It is based on complete mathematical analysis of bipolar transistor based on its improved base dynamics solution $n_{m}(x, \tau)=\nu_{m}(x) \Upsilon^{(m)}(\tau)$ of electron concentration description in [9]. It uses non-linear higher order current controlled sources in electrical circuit representation.

\subsection{Numerical Model}

This model solves the physical system, which is in the form of coupled partial differential equations. It uses Finite Difference Method, Gauss Elimination Method and Simpson's Method to solve these equations and to determine the terminal currents. It also computes minority carrier mobilities and life times based on Shockley Read Hall Recombination Mechanism.

On the basis of current density equation in one dimension and carrier flow described by the continuity equation, we can write this 
equation, which represents the carrier flow as

$$
\frac{\partial n}{\partial t}=D_{n} \frac{\partial^{2} n}{\partial x^{2}}-\frac{n}{\tau_{n}}
$$

where excess concentration $n=n_{t}-n_{p 0}$.

When we consider the higher order effect, which has been discussed by Sipila et al. [9] we can write excess minority concentration in the following form: $n_{m}(x, t)=\sqrt{ }_{m} \gamma^{m}(\tau)$

(1) when $m=0$ : zero order effect

(2) $>0$ : higher order effects

$$
\begin{aligned}
& \frac{\partial n}{\partial t}=D_{n} \frac{\partial^{2} n}{\partial x^{2}}-\frac{n}{\tau_{n}} \\
& \frac{\partial^{2} \nu_{0}}{\partial x^{2}}-\frac{\nu_{0}}{L_{n}^{2}}=0 \\
& \frac{\partial^{2} \nu_{1}}{\partial x^{2}}-\frac{\nu_{1}}{L_{n}^{2}}=\frac{\nu_{0}}{D_{n}} \\
& \frac{\partial \nu_{3}}{\partial X^{2}}-\frac{\nu_{3}}{L_{n}^{2}}=\frac{\nu_{2}}{D_{n}}
\end{aligned}
$$

For zero order solution, boundary condition is

$$
\begin{aligned}
& \nu_{0}(0)=n_{p 0} \\
& \nu_{0}(W)=0
\end{aligned}
$$

and for higher order effect boundary condition is

$$
\nu_{m}(0)=\nu_{m}(w)=0
$$

We integrated these position dependent functions in neutral base region to calculate the following coefficients used in terminal current calculations:

$$
\begin{aligned}
& a_{0}=\int_{0}^{w} \nu_{0}(x) d x \\
& a_{1}=\int_{0}^{w} \nu_{1}(x) d x \\
& a_{2}=\int_{0}^{w} \nu_{2}(x) d x \\
& a_{3}=\int_{0}^{w} \nu_{3}(x) d x
\end{aligned}
$$


Now we can use these steps to find out emitter current at $x=0$ of neutral base region.

$$
\begin{aligned}
& i_{m}(x, t)=-A_{q} D_{n} \frac{\partial n_{m}}{\partial x}(x, t) \\
& i_{e 0}(x, t)=-A_{q} D_{n} \nu_{0}^{\prime}(x) \gamma(t) \\
& i_{e 1}(x, t)=-A_{q} D_{n} \nu_{1}^{\prime}(x) \gamma^{\prime}(t) \\
& i_{e 2}(x, t)=-A_{q} D_{n} \nu_{2}^{\prime}(x) \gamma^{\prime \prime}(t) \\
& i_{e 3}(x, t)=-A_{q} D_{n} \nu_{3}^{\prime}(x) \gamma^{\prime \prime \prime}(t)
\end{aligned}
$$

Similarly we can compute collector current at $x=w$

$$
\begin{aligned}
& i_{c 0}(x, t)=-A_{q} D_{n} \nu_{0}^{\prime}(x) \gamma(t) \\
& i_{c 1}(x, t)=-A_{q} D_{n} \nu_{1}^{\prime}(x) \gamma^{\prime}(t) \\
& i_{c 2}(x, t)=-A_{q} D_{n} \nu_{2}^{\prime}(x) \gamma^{\prime \prime}(t) \\
& i_{c 3}(x, t)=-A_{q} D_{n} \nu_{3}^{\prime}(x) \gamma^{\prime \prime \prime}(t)
\end{aligned}
$$

We used the expression discussed by Sipila et al. [9] to compute the base current:

$$
\begin{aligned}
i_{b}(t)=A_{q} & {\left[\frac{a_{0}}{\tau_{n}} \gamma(t)+\left(a_{0}+\frac{a_{1}}{\tau_{n}}\right) \gamma^{\prime}(t)\right.} \\
& \left.+\left(a_{1}+\frac{a_{2}}{\tau_{n}}\right) \gamma^{\prime \prime}(t)+\left(a_{2}+\frac{a_{3}}{\tau_{n}}\right) \gamma^{\prime \prime \prime}(t)\right]
\end{aligned}
$$

\subsection{Lumped Transistor Model}

The model is based on the solution of partial differential equations, which yield network like models of a device. Since a physical device operates by transmitting information between points in space, this information always results in delays. If we are to model such a device completely we must therefore include time and space variables in our characterization. Let us define [10]

$S \triangleq q . A . \Delta x$ ("Storance", representing charge storage in the $n$th lump)

$H^{d} \triangleq q . A . D / \Delta x$ ("Diffusance", represents diffusion between the $n$th lump and the adjacent lump)

$H^{c} \triangleq q . A . \Delta x / \tau_{n}=$ ("Combinance", represents recombination within $n$th lump) 
A transistor can be formed by two diodes in close proximity, where a single lump model of emitter and collector regions is used. When these lumps are reflected to the base by "Voltage" scaling of the carrier density levels, and when the base is represented by a single lump model, the equivalent circuit has the form shown in Figure 1.

The parameters can be expressed as:

$$
\begin{aligned}
H^{d} & =\frac{q A D_{b}}{W} \\
\tilde{H}_{p_{e}} & =\frac{q A L_{e}}{\tau_{e}} \cdot \frac{p_{e 0}}{n_{b 0}} ; \tilde{H}_{n_{n}}=\frac{q A W}{2 \tau_{b}} ; \tilde{H}_{p_{c}}=\frac{q A L_{c}}{\tau_{c}} \cdot \frac{p_{c 0}}{n_{b 0}} ; \\
\tilde{S}_{p_{e}} & =\frac{q A L_{e}}{2} \cdot \frac{p_{e 0}}{n_{b 0}} ; \tilde{S}_{n_{e}}=\frac{q A W}{2} ; \tilde{S}_{p_{c}}=\frac{q A L_{c}}{2} \cdot \frac{p_{c 0}}{n_{b 0}} \\
\tilde{H}_{n_{c}} & =\frac{q A W}{2 \tau_{b}} ; \tilde{S}_{n e}=\frac{q A W}{2}
\end{aligned}
$$

\subsubsection{Large Signal Dynamic Model}

Now the differential equations describing the model shown in Figure 19 are given by:

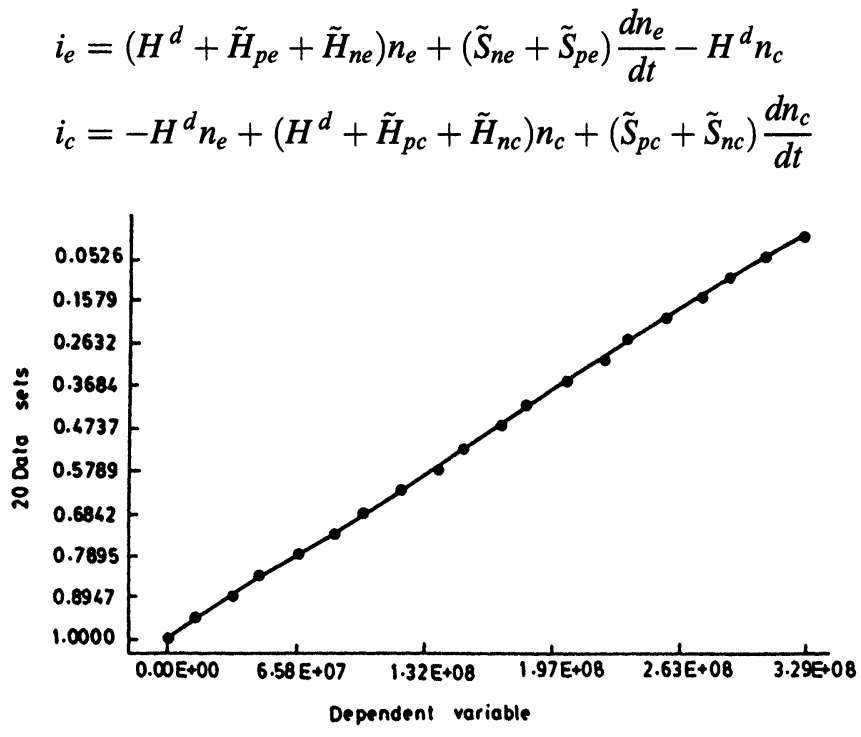

FIGURE 1 Gamma 0 vs. $X / W$. 


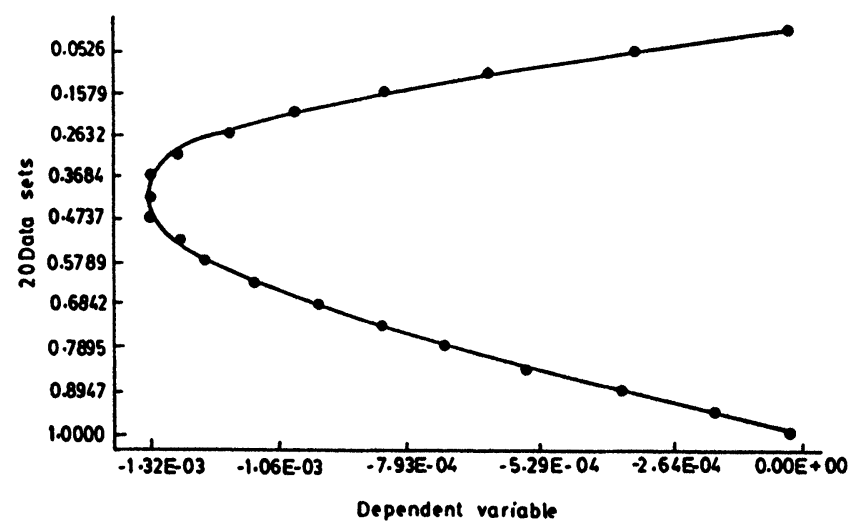

FIGURE 2 Gamma 1 vs. $X / W$.

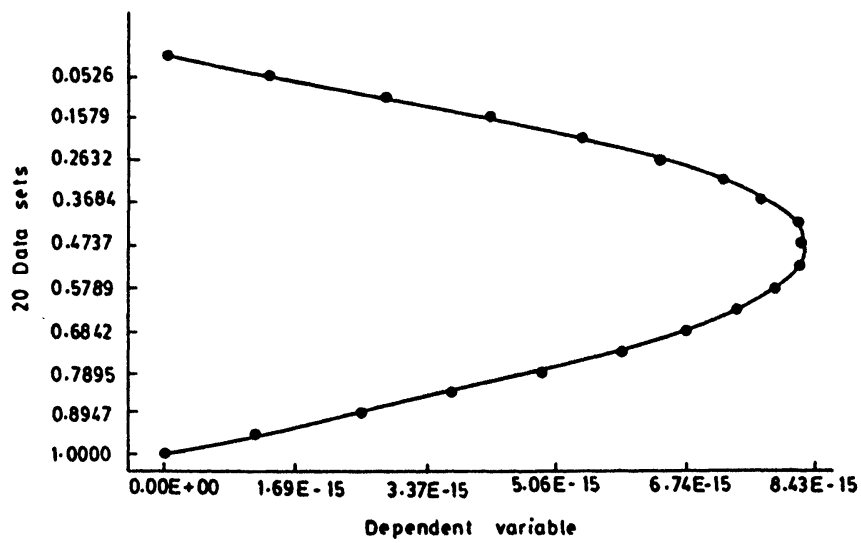

FIGURE 3 Gamma 2 vs. $X / W$.

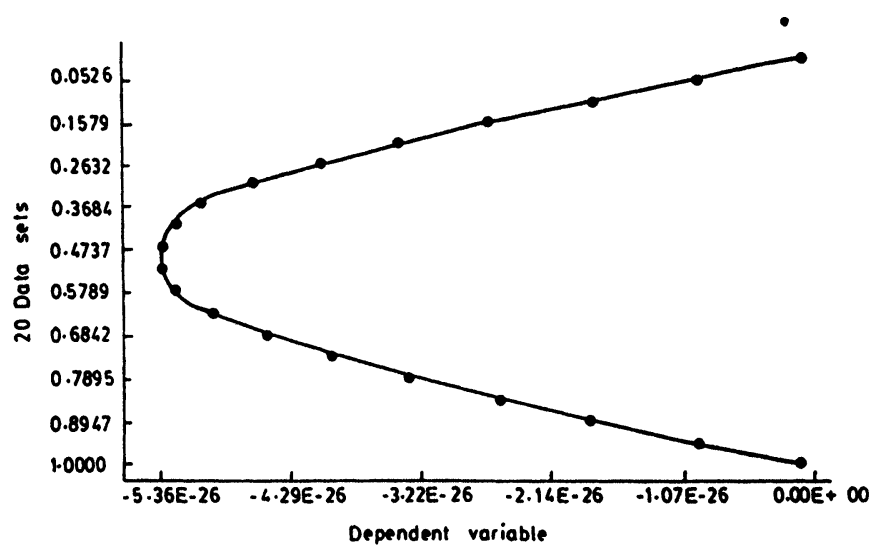

FIGURE 4 Gamma 3 vs. $X / W$. 


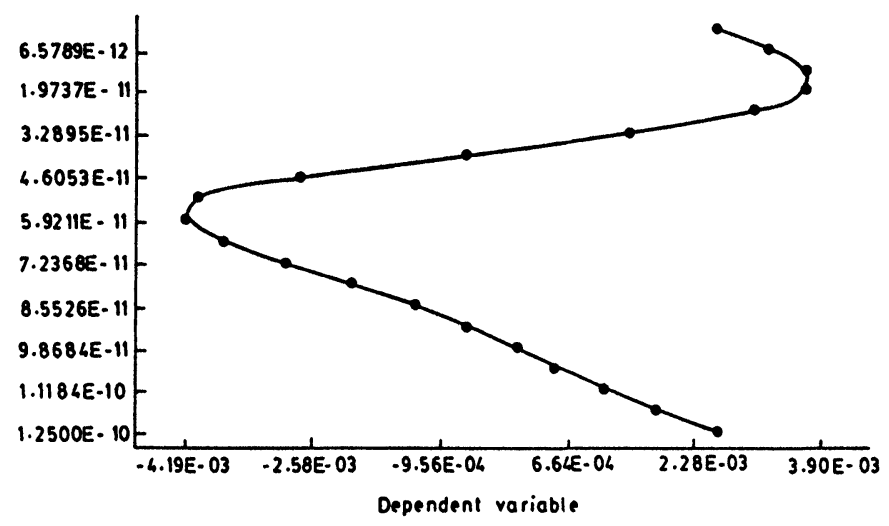

FIGURE 5 IB(Amp) vs. time (sec).

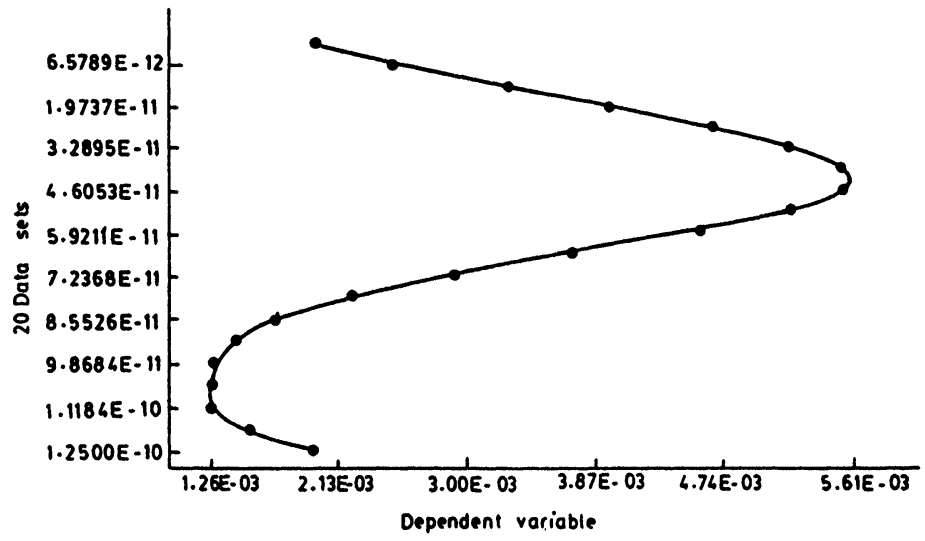

FIGURE 6 IC(Amp) vs. time (sec).

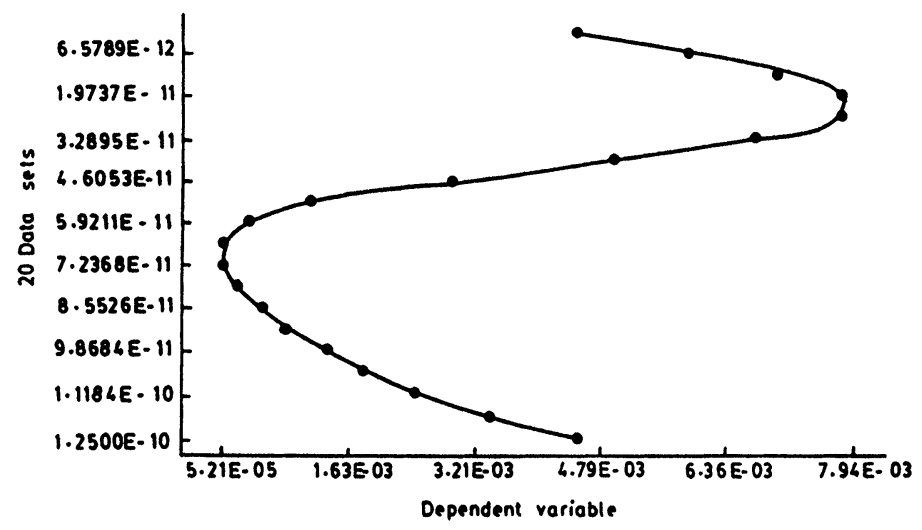

FIGURE 7 IE(Amp) vs. time (sec). 


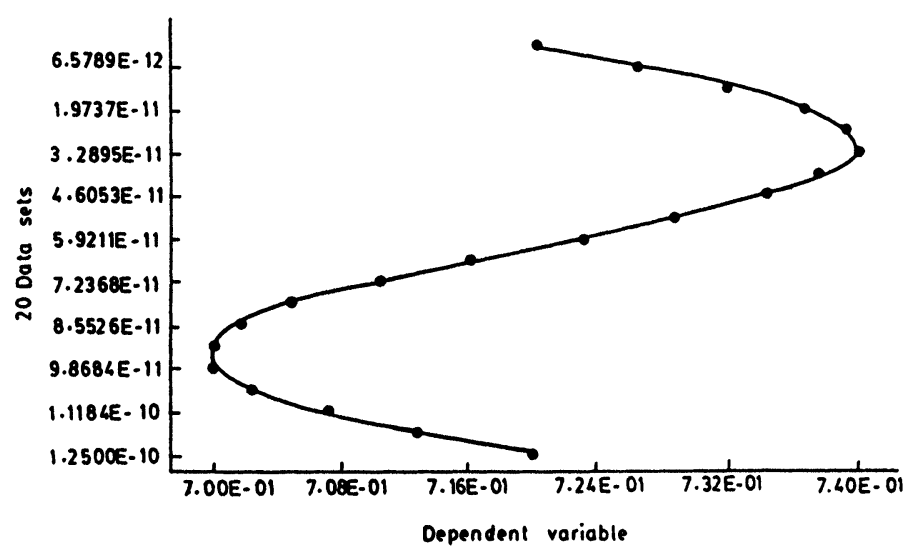

FIGURE 8 Input(Volts) vs. time (sec).

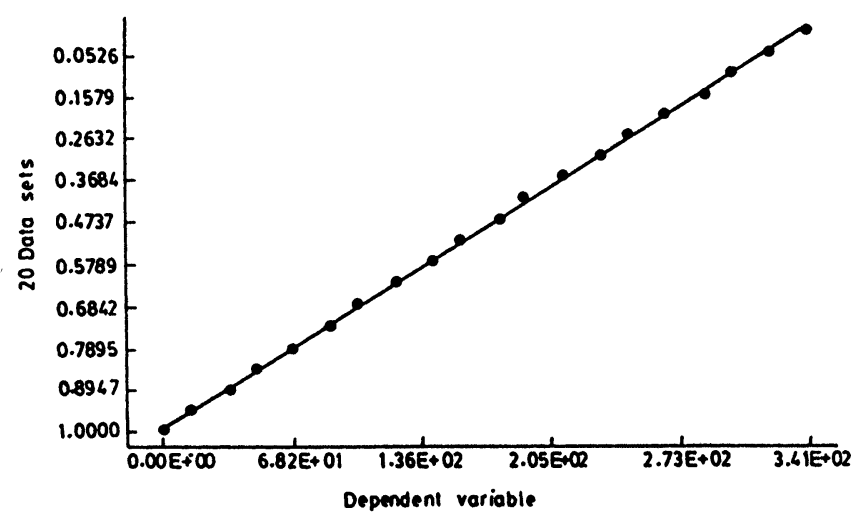

FIGURE 9 Gamma 0 vs. $X / W$.

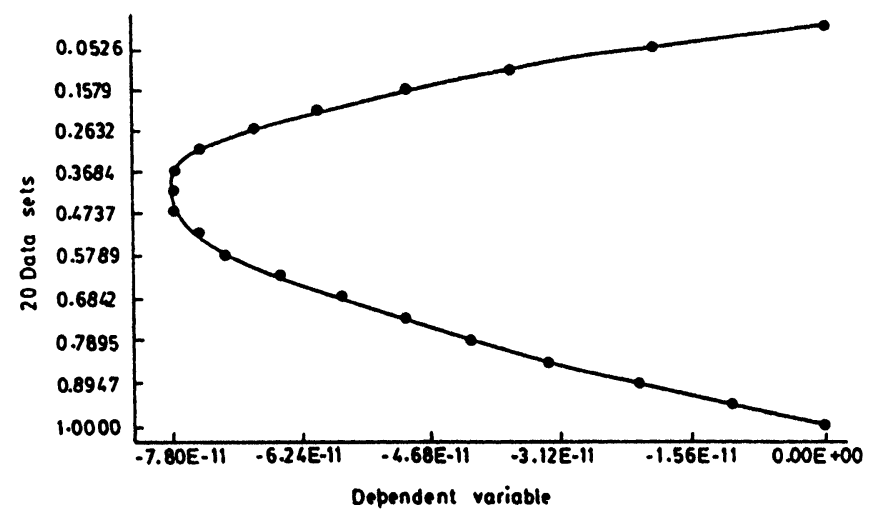

FIGURE 10 Gamma 1 vs. $X / W$. 


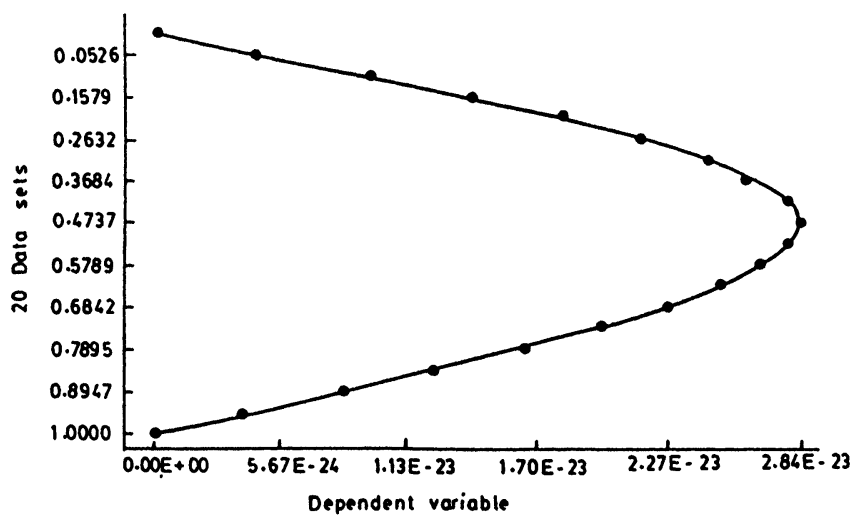

FIGURE 11 Gamma 2 vs. $X / W$.

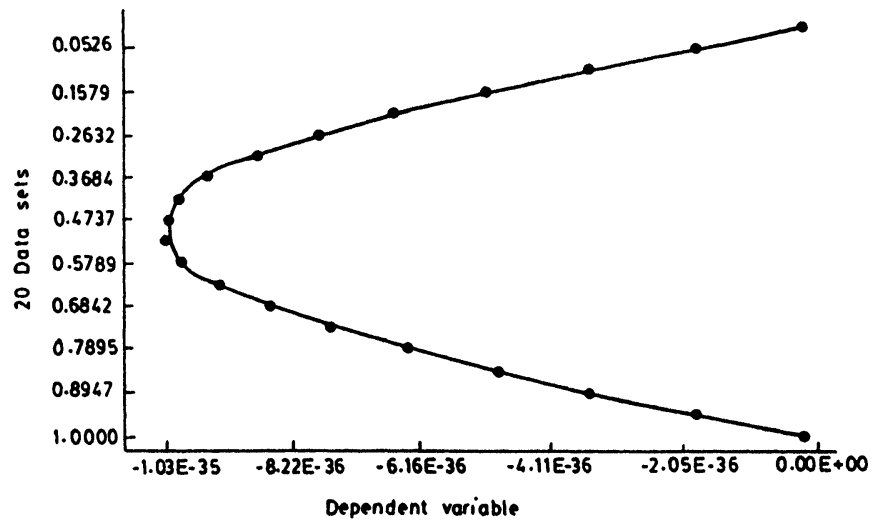

FIGURE 12 Gamma 3 vs. $X / W$.

Since these are linear equations, they do not include non linearity of the junction law.

$$
\begin{aligned}
i_{e}= & \left(H^{d}+\tilde{H}_{p e}+\tilde{H}_{n e}\right) n_{b 0}\left(\mathrm{e}^{V_{e b} / V_{T}}-1\right) \\
& +\left(\tilde{S}_{n e}+\tilde{S}_{p e}\right)\left(n_{b 0} / V_{T}\right) \mathrm{e}^{V_{\mathrm{eb}} / V_{T}} \frac{d V_{e b}}{d t}-H^{d} n_{b 0}\left(\mathrm{e}^{V_{c b} / V_{T}}-1\right)
\end{aligned}
$$



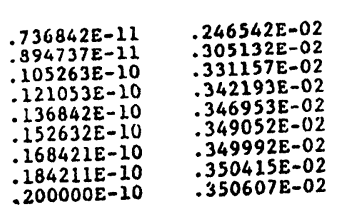
$.142936 \mathrm{E}+02$
$.999242 \mathrm{E}+01$ $.784667 E+0$ $757958 E+0$ $.746109 \mathrm{E}+0$ $740785 E+0$

$.738380 \mathrm{E}+0$

$.350415 E-02 \quad .737290 \mathrm{E}+01$

$.321485 \mathrm{E}-12$

$.459867 \mathrm{E}-12$

$.542787 \mathrm{E}-12$

$.585622 \mathrm{E}-12$

$.606258 \mathrm{E}-12$

$615886 \mathrm{E}-12$

$.620312 \mathrm{E}-12$

$.184211 \mathrm{E}-10$

$.623253 E-12$

20Data Sets

$-1.0000 \mathrm{E}-11$

$-1.0000 \mathrm{E}-11$
$-8.4211 \mathrm{E}-12$

$-8.4211 \mathrm{E}-12$

$5.8421 \mathrm{E}-12$

$-3.6842 \mathrm{E}-12$

$-2.1053 \mathrm{E}-12$

$-5.2632 \mathrm{E}-13$

$1.0526 \mathrm{E}-12$

$2.6316 \mathrm{E}-12$

4.2105E-12

$5.7895 \mathrm{E}-12$

$7.3684 \mathrm{E}-12$

$8.9474 \mathrm{E}-12$

$1.0526 \mathrm{E}-11$

$1.2105 \mathrm{E}-11$

$1.3684 \mathrm{E}-1$

$1.5263 \mathrm{E}-11$

$1.6842 \mathrm{E}-11$

$1.8421 E-11$

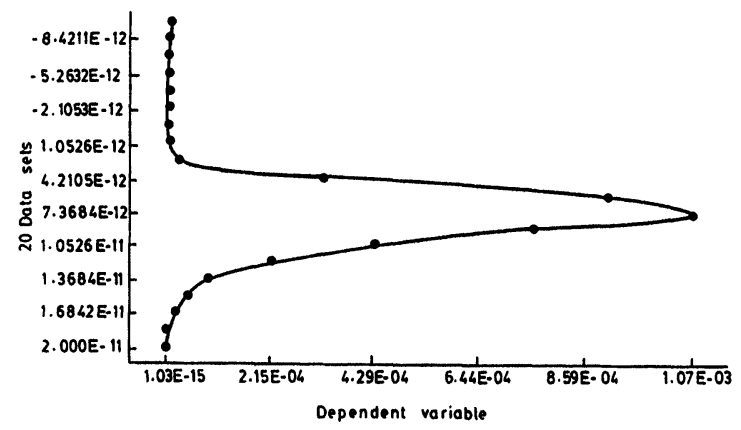

FIGURE 13 IB(Amp) vs. time (sec).

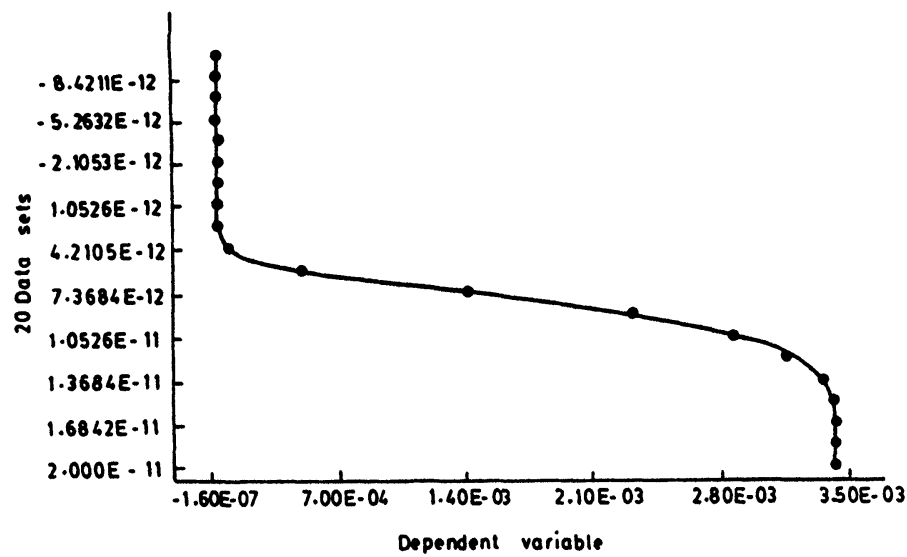

FIGURE 14 IC(Amp) vs. time (sec). 


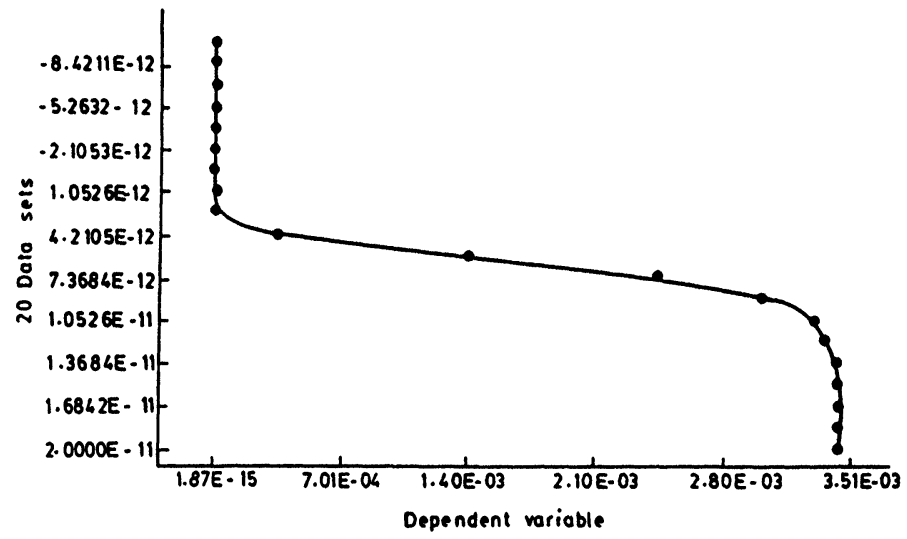

FIGURE 15 IE(Amp) vs. time (sec).

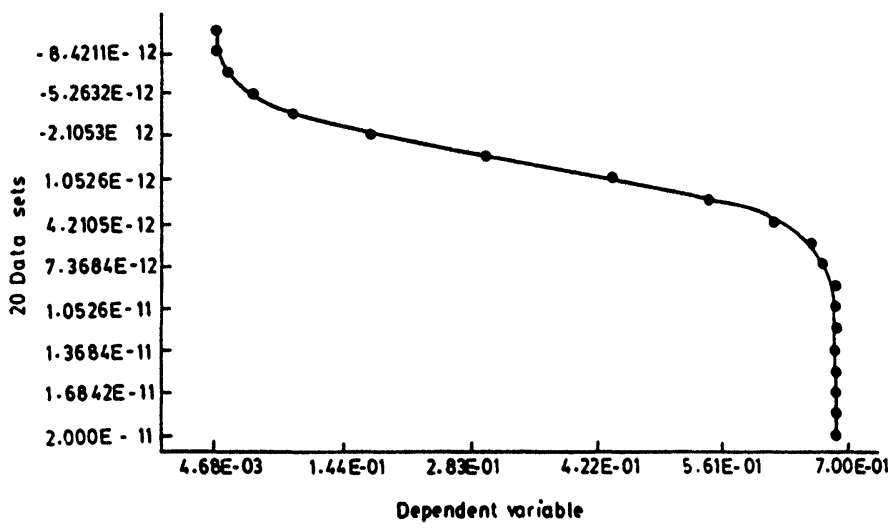

FIGURE 16 Input(Volt) vs. time (sec).

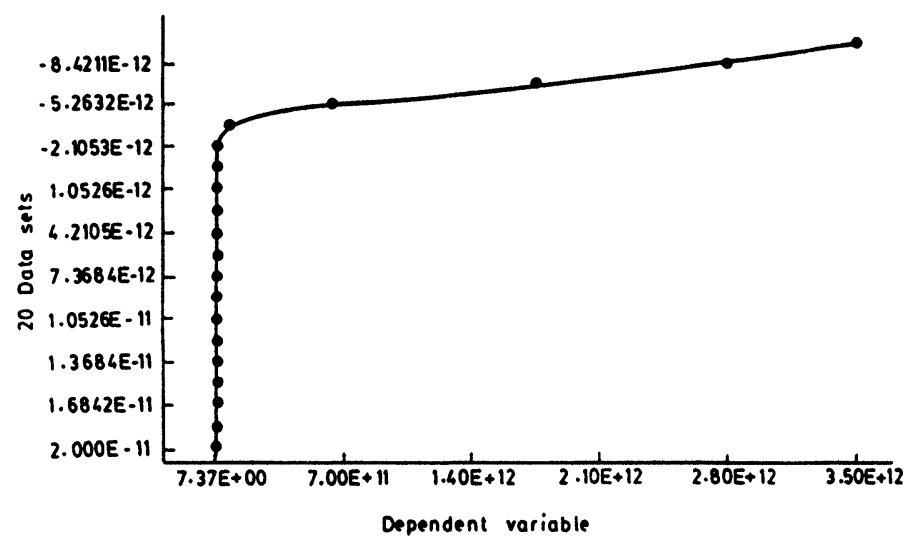

FIGURE 17 Time(sec) vs. $\operatorname{Re}(\mathrm{Ohms})$. 


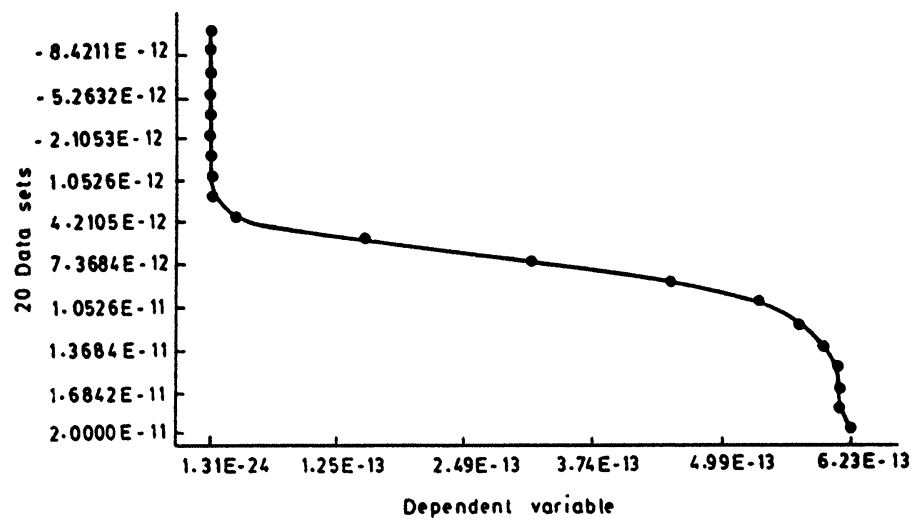

FIGURE 18 Time(sec) vs. Ce (F).

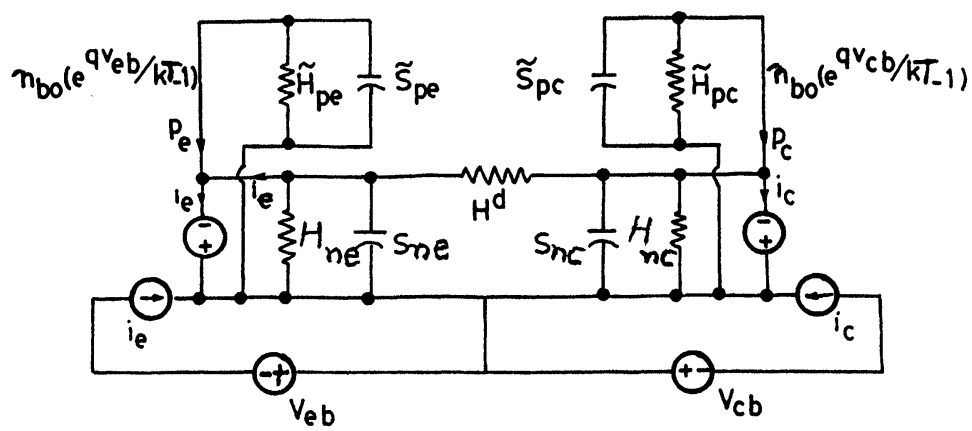

FIGURE 19

$$
\begin{aligned}
i_{c}= & -H^{d} n_{b 0}\left(\mathrm{e}^{V_{e b} / V_{T}}-1\right)+\left(H^{d}+\tilde{H}_{p c}+\tilde{H}_{n c}\right) n_{b 0}\left(\mathrm{e}^{V_{c b} / V_{T}}-1\right) \\
& +\left(\tilde{S}_{n c}+\tilde{S}_{p c}\right)\left(n_{b 0} / V_{T}\right) \mathrm{e}^{V_{c b} / V_{T}} \frac{d V_{c b}}{d t}
\end{aligned}
$$

Although the model of Figure 19 is a large signal dynamic one, we will convert it to a more common form. Figure 19 is described on the basis of Eqs. (11) and (12), if the capacitors are described by an equation of the from $i=c(t) d v / d t$ and the elements have the value of column three of Table I given below. 


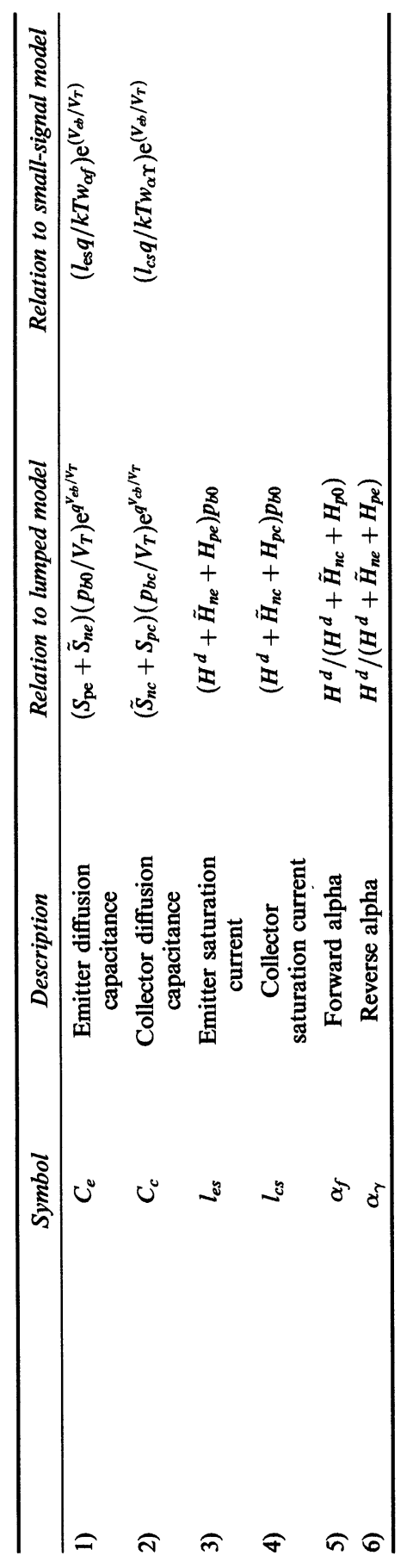


TABLE I Input to the program is area of transistor $=4.020000 \mathrm{E}-09$ width $=$ $2.500000 \mathrm{E}-07$ minority carrier concentration $=3.300000 \mathrm{E}+08$ Life time $=3.000000$ $\mathrm{E}-09$ diffusion constant $=1.000000 \mathrm{E}-03$ diffusion length $=1.732051 \mathrm{E}-06$ small signal analysis of the input function $V(T)=C 1+C 2 * \sin (2 * P I * F * T)$ where $C 1=$ $7.20 \mathrm{E}-01 C 2=2.00 \mathrm{E}-02$ frequency $=8.00 \mathrm{E}+09$

\begin{tabular}{lcccr}
\hline$x$ & Gamma 0 & \multicolumn{1}{c}{ Gamma 1} & \multicolumn{1}{c}{ Gamma_2 } & \multicolumn{1}{c}{ Gamma 3} \\
\hline .00 & $.3288570 \mathrm{E}+09$ & $.0000000 \mathrm{E}+00$ & $.0000000 \mathrm{E}+00$ & $.0000000 \mathrm{E}+00$ \\
.05 & $.3115487 \mathrm{E}+09$ & $-.3331204 \mathrm{E}-03$ & $.1503230 \mathrm{E}-14$ & $-.8999306 \mathrm{E}-26$ \\
.11 & $.2942404 \mathrm{E}+09$ & $-.6122831 \mathrm{E}-03$ & $.2948700 \mathrm{E}-14$ & $-.1773784 \mathrm{E}-25$ \\
.16 & $.2769322 \mathrm{E}+09$ & $-.8404686 \mathrm{E}-03$ & $.4287996 \mathrm{E}-14$ & $-.2596484 \mathrm{E}-25$ \\
.21 & $.2596239 \mathrm{E}+09$ & $-.1020660 \mathrm{E}-02$ & $.5481533 \mathrm{E}-14$ & $-.3344796 \mathrm{E}-25$ \\
.26 & $.2423156 \mathrm{E}+09$ & $-.1155844 \mathrm{E}-02$ & $.6498047 \mathrm{E}-14$ & $-.3998012 \mathrm{E}-25$ \\
.32 & $.2250074 \mathrm{E}+09$ & $-.1249009 \mathrm{E}-02$ & $.7314074 \mathrm{E}-14$ & $-.4538497 \mathrm{E}-25$ \\
.37 & $.2076991 \mathrm{E}+09$ & $-.1303147 \mathrm{E}-02$ & $.7913439 \mathrm{E}-14$ & $-.4952093 \mathrm{E}-25$ \\
.42 & $.1903909 \mathrm{E}+09$ & $-.1321251 \mathrm{E}-02$ & $.8286733 \mathrm{E}-14$ & $-.5228396 \mathrm{E}-25$ \\
.47 & $.1730826 \mathrm{E}+09$ & $-.1306316 \mathrm{E}-02$ & $.8430802 \mathrm{E}-14$ & $-.5360930 \mathrm{E}-25$ \\
.53 & $.1557744 \mathrm{E}+09$ & $-.1261339 \mathrm{E}-02$ & $.8348222 \mathrm{E}-14$ & $-.5347193 \mathrm{E}-25$ \\
.58 & $.1384661 \mathrm{E}+09$ & $-.1189321 \mathrm{E}-02$ & $.8046784 \mathrm{E}-14$ & $-.5188614 \mathrm{E}-25$ \\
.63 & $.1211578 \mathrm{E}+09$ & $-.1093262 \mathrm{E}-02$ & $.7538975 \mathrm{E}-14$ & $-.4890421 \mathrm{E}-25$ \\
.68 & $.1038496 \mathrm{E}+09$ & $-.9761631 \mathrm{E}-03$ & $.6841455 \mathrm{E}-14$ & $-.4461425 \mathrm{E}-25$ \\
.74 & $.8654131 \mathrm{E}+08$ & $-.8410288 \mathrm{E}-03$ & $.5974536 \mathrm{E}-14$ & $-.3913724 \mathrm{E}-25$ \\
.79 & $.6923304 \mathrm{E}+08$ & $-.6908631 \mathrm{E}-03$ & $.4961667 \mathrm{E}-14$ & $-.3262362 \mathrm{E}-25$ \\
.84 & $.5192479 \mathrm{E}+08$ & $-.5286711 \mathrm{E}-03$ & $.3828901 \mathrm{E}-14$ & $-.2524909 \mathrm{E}-25$ \\
.89 & $.3461654 \mathrm{E}+08$ & $-.3574590 \mathrm{E}-03$ & $.2604385 \mathrm{E}-14$ & $-.1721021 \mathrm{E}-25$ \\
.95 & $.1730827 \mathrm{E}+08$ & $-.1802330 \mathrm{E}-03$ & $.1317833 \mathrm{E}-14$ & $-.8719433 \mathrm{E}-26$ \\
1.00 & $.0000000 \mathrm{E}+00$ & $.0000000 \mathrm{E}+00$ & $.0000000 \mathrm{E}+00$ & $.0000000 \mathrm{E}+00$ \\
\hline
\end{tabular}

\subsubsection{Small Signal Model}

The small signal model [10] Figure 21 follows the large signal model by replacing each element by its small signal equivalent. For example, the emitter diode is replaced by the linear resistor:

$$
R_{e}^{0}=\frac{1}{G_{e}^{0}} \triangleq V_{T} /\left(I_{e s} \mathrm{e}^{V_{e b} / V_{T}}\right)
$$

and the small signal emitter diffusion capacitor is

$$
C_{e}^{0}=\left(S_{p e}+\tilde{S}_{n e}\right) \frac{\tilde{p}_{b 0}}{V_{T}} \mathrm{e}^{V_{e b}^{0} / V_{T}}
$$




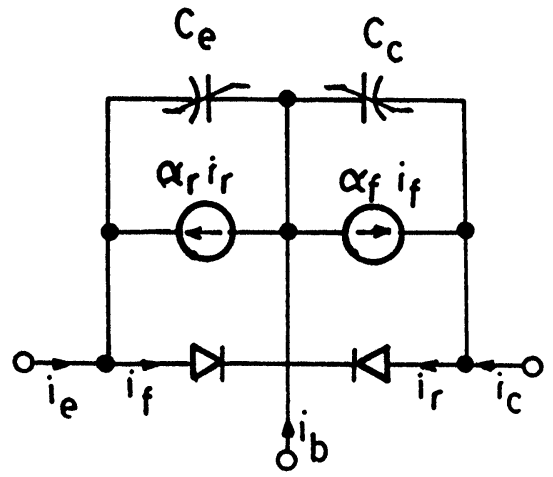

FIGURE 20
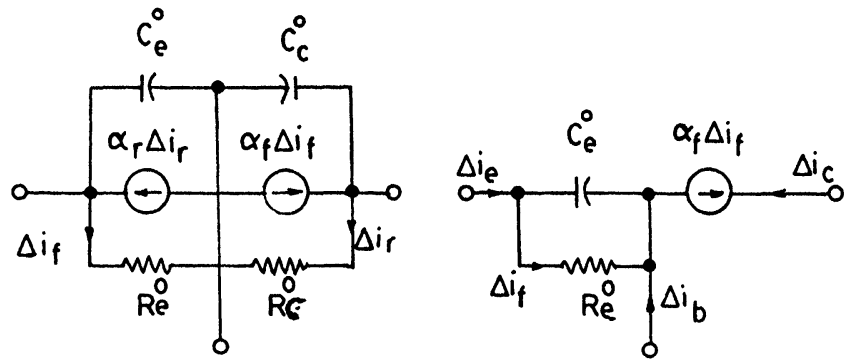

FIGURE 21

When the transistor is forward biased then

$$
\frac{\Delta I_{c}}{\Delta I_{e}}=\frac{\alpha_{f}}{1+R_{e}^{0} C_{e}^{0} S}=\frac{\alpha_{f}}{1+\frac{\left(S_{p e}+\tilde{S}_{n e}\right) p_{b 0} S}{I_{e s}}}=\frac{\alpha_{f}}{1+s / w_{\alpha f}}
$$

where $\quad 1 / \omega_{\alpha f}=\frac{\left(S_{p e}+\tilde{S}_{n e}\right) P_{b 0}}{I_{e s}}$

From the circuit shown in Figure 20, $W$ is measurable as the 3- $d B$ cutoff frequency in the forward direction. The large signal capacitance [10] of table can now be written as:

$$
\begin{aligned}
C_{e} & =\frac{\left(S_{n e}+\tilde{S}_{p e}\right) n_{b 0}}{V_{T}} \mathrm{e}^{V_{e b} / V_{T}}=\frac{q I_{e s} \mathrm{e}^{V_{e b} / V_{T}}}{V_{T} \omega_{\alpha f}} \\
& \cong q I_{e} / K T \omega_{\alpha f}
\end{aligned}
$$


TABLE II It uses more accurate model for analysis sinusoidal analysis

\begin{tabular}{lccc}
\hline \multicolumn{4}{c}{ Result } \\
\hline Time $(\mathrm{Sec})$ & $I B($ Amp $)$ & $I C(\mathrm{Amp})$ & $I E(\mathrm{Amp})$ \\
\hline $.000000 \mathrm{E}+00$ & $.262804 \mathrm{E}-02$ & $.197111 \mathrm{E}-02$ & $.459914 \mathrm{E}-02$ \\
$.657895 \mathrm{E}-11$ & $.335620 \mathrm{E}-02$ & $.255482 \mathrm{E}-02$ & $.591101 \mathrm{E}-02$ \\
$.131579 \mathrm{E}-10$ & $.386845 \mathrm{E}-02$ & $.327862 \mathrm{E}-02$ & $.714707 \mathrm{E}-02$ \\
$.197368 \mathrm{E}-10$ & $.390296 \mathrm{E}-02$ & $.403765 \mathrm{E}-02$ & $.794061 \mathrm{E}-02$ \\
$.263158 \mathrm{E}-10$ & $.318003 \mathrm{E}-02$ & $.471859 \mathrm{E}-02$ & $.789862 \mathrm{E}-02$ \\
$.328947 \mathrm{E}-10$ & $.158183 \mathrm{E}-02$ & $.525192 \mathrm{E}-02$ & $.683374 \mathrm{E}-02$ \\
$.394737 \mathrm{E}-10$ & $-.601052 \mathrm{E}-03$ & $.558003 \mathrm{E}-02$ & $.497898 \mathrm{E}-02$ \\
$.460526 \mathrm{E}-10$ & $-.267187 \mathrm{E}-02$ & $.560591 \mathrm{E}-02$ & $.293403 \mathrm{E}-02$ \\
$.526316 \mathrm{E}-10$ & $-.394544 \mathrm{E}-02$ & $.525212 \mathrm{E}-02$ & $.130668 \mathrm{E}-02$ \\
$.592105 \mathrm{E}-10$ & $-.419464 \mathrm{E}-02$ & $.456816 \mathrm{E}-02$ & $.373521 \mathrm{E}-03$ \\
$.657895 \mathrm{E}-10$ & $-.367485 \mathrm{E}-02$ & $.372696 \mathrm{E}-02$ & $.521126 \mathrm{E}-04$ \\
$.723684 \mathrm{E}-10$ & $-.281269 \mathrm{E}-02$ & $.291716 \mathrm{E}-02$ & $.104466 \mathrm{E}-03$ \\
$.789474 \mathrm{E}-10$ & $-.192986 \mathrm{E}-02$ & $.225561 \mathrm{E}-02$ & $.325748 \mathrm{E}-03$ \\
$.855263 \mathrm{E}-10$ & $-.116692 \mathrm{E}-02$ & $.177703 \mathrm{E}-02$ & $.610113 \mathrm{E}-03$ \\
$.921053 \mathrm{E}-10$ & $-.533342 \mathrm{E}-03$ & $.146789 \mathrm{E}-02$ & $.934549 \mathrm{E}-03$ \\
$.986842 \mathrm{E}-10$ & $.233907 \mathrm{E}-04$ & $.130172 \mathrm{E}-02$ & $.132512 \mathrm{E}-02$ \\
$.105263 \mathrm{E}-09$ & $.572875 \mathrm{E}-03$ & $.126046 \mathrm{E}-02$ & $.183333 \mathrm{E}-02$ \\
$.111842 \mathrm{E}-09$ & $.117699 \mathrm{E}-02$ & $.134387 \mathrm{E}-02$ & $.252085 \mathrm{E}-02$ \\
$.118421 \mathrm{E}-09$ & $.186895 \mathrm{E}-02$ & $.157113 \mathrm{E}-02$ & $.344008 \mathrm{E}-02$ \\
$.125000 \mathrm{E}-09$ & $.262804 \mathrm{E}-02$ & $.197111 \mathrm{E}-02$ & $.459914 \mathrm{E}-02$ \\
\hline
\end{tabular}

TABLE III Input to the program is Area of transistor $=4.020000 \mathrm{E}-05$ Width $=$ $1.000000 \mathrm{E}-05$ Minority carrier Concentration (Base) $=340.909100$ Base doping concentration $=6.600000 \mathrm{E}+17$ Emitter doping concentration $=1.000000 \mathrm{E}+19$ Collector doping concentration $=1.000000 \mathrm{E}+16$ In Base $\rightarrow$ Life time $=7.042254 \mathrm{E}-08$ Diffusion constant $=28.028600$ Diffusion Length $=1.404936-03$. Small signal analysis of the input function $v(t)=\mathrm{E} 0 /[1+\exp (-\mathrm{kt})]$ where $\mathrm{E} 0=7.00 \mathrm{E}-01$ Damping factor $(\mathrm{K})=5.000000 \mathrm{E}+11$ Transient start time $=-10 \mathrm{psec}$ stop time $=20 \mathrm{psec}$ $\mathrm{VCB}=0.000000 \mathrm{E}+00$. Ies $(\mathrm{Amp})=6.161405 \mathrm{E}-15 \mathrm{Ics}(\mathrm{Amp})=6.363502 \mathrm{E}-15 \mathrm{Col}-$ lector Capacitance $(\mathrm{F})=3.368963 \mathrm{E}-21 \mathrm{hFE}=883.191000$ Break down voltage $\mathrm{BVbe}=2.306128 \mathrm{BVcb}=53.4000003-\mathrm{db}$ cut off frequency for Waf $=2.176192 \mathrm{E}+11$ $\mathrm{War}=7.304179 \mathrm{E}+07$

\begin{tabular}{lcccr}
\hline$x$ & Gamma_0 & Gamma_ & Gamma_2 & \multicolumn{1}{c}{ Gamma_3 } \\
\hline .00 & $.3409063 \mathrm{E}+03$ & $.0000000 \mathrm{E}+00$ & $.0000000 \mathrm{E}+00$ & $.0000000 \mathrm{E}+00$ \\
.05 & $.3229638 \mathrm{E}+03$ & $-.1968327 \mathrm{E}-10$ & $.5057831 \mathrm{E}-23$ & $-.1724555 \mathrm{E}-35$ \\
.11 & $.3050214 \mathrm{E}+03$ & $-.3617466 \mathrm{E}-10$ & $.9921130 \mathrm{E}-23$ & $-.3399122 \mathrm{E}-35$ \\
.16 & $.2870789 \mathrm{E}+03$ & $-.4965150 \mathrm{E}-10$ & $.1442691 \mathrm{E}-22$ & $-.4975638 \mathrm{E}-35$ \\
.21 & $.2691365 \mathrm{E}+03$ & $-.6029112 \mathrm{E}-10$ & $.1844198 \mathrm{E}-22$ & $-.6409572 \mathrm{E}-35$ \\
.26 & $.2511941 \mathrm{E}+03$ & $-.6827084 \mathrm{E}-10$ & $.2186119 \mathrm{E}-22$ & $-.7661241 \mathrm{E}-35$ \\
.32 & $.2332516 \mathrm{E}+03$ & $-.7376798 \mathrm{E}-10$ & $.2460567 \mathrm{E}-22$ & $-.8696855 \mathrm{E}-35$ \\
.37 & $.2153092 \mathrm{E}+03$ & $-.7695988 \mathrm{E}-10$ & $.2662110 \mathrm{E}-22$ & $-.9489289 \mathrm{E}-35$ \\
.42 & $.1973668 \mathrm{E}+03$ & $-.7802386 \mathrm{E}-10$ & $.2787592 \mathrm{E}-22$ & $-.1001862 \mathrm{E}-34$ \\
.47 & $.1794243 \mathrm{E}+03$ & $-.7713724 \mathrm{E}-10$ & $.2835962 \mathrm{E}-22$ & $-.1027246 \mathrm{E}-34$ \\
.53 & $.1614819 \mathrm{E}+03$ & $-.7447735 \mathrm{E}-10$ & $.2808097 \mathrm{E}-22$ & $-.1024601 \mathrm{E}-34$ \\
.58 & $.1435395 \mathrm{E}+03$ & $-.7022151 \mathrm{E}-10$ & $.2706625 \mathrm{E}-22$ & $-.9942033 \mathrm{E}-35$ \\
.63 & $.1255970 \mathrm{E}+03$ & $-.6454705 \mathrm{E}-10$ & $.2535753 \mathrm{E}-22$ & $-.9370560 \mathrm{E}-35$ \\
.68 & $.1076546 \mathrm{E}+03$ & $-.5763131 \mathrm{E}-10$ & $.2301088 \mathrm{E}-22$ & $-.8548475 \mathrm{E}-35$ \\
.74 & $.8971217 \mathrm{E}+02$ & $-.4965159 \mathrm{E}-10$ & $.2009465 \mathrm{E}-22$ & $-.7498970 \mathrm{E}-35$ \\
.79 & $.7176973 \mathrm{E}+02$ & $-.4078524 \mathrm{E}-10$ & $.1668770 \mathrm{E}-22$ & $-.6250868 \mathrm{E}-35$ \\
\hline
\end{tabular}


TABLE III (Continued)

\begin{tabular}{lcccr}
\hline$x$ & Gamma_0 & \multicolumn{1}{c}{ Gamma 1} & Gamma_2 & \multicolumn{1}{c}{ Gamma_3 } \\
\hline .84 & $.5382730 \mathrm{E}+02$ & $-.3120957 \mathrm{E}-10$ & $.1287768 \mathrm{E}-22$ & $-.4837839 \mathrm{E}-35$ \\
.89 & $.3588488 \mathrm{E}+02$ & $-.2110193 \mathrm{E}-10$ & $.8759205 \mathrm{E}-23$ & $-.3297539 \mathrm{E}-35$ \\
.95 & $.1794242 \mathrm{E}+02$ & $-.1063963 \mathrm{E}-10$ & $.4432179 \mathrm{E}-23$ & $-.1670672 \mathrm{E}-35$ \\
1.00 & $.0000000 \mathrm{E}+00$ & $.0000000 \mathrm{E}+00$ & $.0000000 \mathrm{E}+00$ & $.0000000 \mathrm{E}+00$ \\
\hline
\end{tabular}

TABLE IV It uses more accurate model for analysis transient analysis

\begin{tabular}{|c|c|c|c|c|}
\hline \multicolumn{5}{|c|}{ Result } \\
\hline Time $(\mathrm{Sec})$ & $I B(A m p)$ & $I C(A m p)$ & $I E(A m p)$ & $\operatorname{Input}(V)$ \\
\hline$-.100000 \mathrm{E}-10$ & $.102589 \mathrm{E}-14$ & $.900817 \mathrm{E}-15$ & $.186564 \mathrm{E}-14$ & $.468500 \mathrm{E}-02$ \\
\hline$-.842105 E-11$ & $.272098 \mathrm{E}-14$ & $.213932 E-14$ & $.469637 \mathrm{E}-14$ & $.102351 \mathrm{E}-01$ \\
\hline$-.684211 E-11$ & $.899365 \mathrm{E}-14$ & $.554955 \mathrm{E}-14$ & $.139910 \mathrm{E}-13$ & $.221508 \mathrm{E}-01$ \\
\hline$-.526316 \mathrm{E}-11$ & $.474588 \mathrm{E}-13$ & $.169357 \mathrm{E}-13$ & $.614508 E-13$ & $.469934 \mathrm{E}-01$ \\
\hline$-.368421 \mathrm{E}-11$ & $.626805 \mathrm{E}-12$ & $.379175 E-13$ & $.628241 \mathrm{E}-12$ & $.957617 \mathrm{E}-01$ \\
\hline$-.210526 \mathrm{E}-11$ & $.334623 E-10$ & $-.537943 E-11$ & $.264816 \mathrm{E}-10$ & $.181104 \mathrm{E}+00$ \\
\hline$-.526316 \mathrm{E}-12$ & $.573740 E-08$ & $-.140509 E-08$ & $.409317 \mathrm{E}-08$ & $.304211 \mathrm{E}+00$ \\
\hline $.105263 \mathrm{E}-11$ & $.867483 E-06$ & $-.159672 E-06$ & $.664368 \mathrm{E}-06$ & $.440036 \mathrm{E}+00$ \\
\hline $.263158 \mathrm{E}-11$ & $.344617 \mathrm{E}-04$ & $.113219 E-05$ & $.332343 E-04$ & $.551936 \mathrm{E}+00$ \\
\hline $.421053 E-11$ & $.328050 \mathrm{E}-03$ & $.901743 E-04$ & $.395905 \mathrm{E}-03$ & $.623990 \mathrm{E}+00$ \\
\hline $.578947 \mathrm{E}-11$ & $.919428 \mathrm{E}-03$ & $.556787 \mathrm{E}-03$ & $.142363 E-02$ & $.663310 \mathrm{E}+00$ \\
\hline $.736842 E-11$ & $.107316 \mathrm{E}-02$ & $.144626 \mathrm{E}-02$ & $.246542 E-02$ & $.682849 E+00$ \\
\hline $.894737 \mathrm{E}-11$ & $.767596 \mathrm{E}-03$ & $.231982 E-02$ & $.305132 \mathrm{E}-02$ & $.692106 \mathrm{E}+00$ \\
\hline $.105263 E-10$ & $.430757 \mathrm{E}-03$ & $.290045 E-02$ & $.331157 \mathrm{E}-02$ & $.696393 E+00$ \\
\hline $.121053 E-10$ & $.215616 \mathrm{E}-03$ & $.321604 \mathrm{E}-02$ & $.342193 E-02$ & $.698358 \mathrm{E}+00$ \\
\hline $.136842 E-10$ & $.102397 \mathrm{E}-03$ & $.337176 \mathrm{E}-02$ & $.346953 \mathrm{E}-02$ & $.699253 \mathrm{E}+00$ \\
\hline $.152632 \mathrm{E}-10$ & $.474930 \mathrm{E}-04$ & $.344521 E-02$ & $.349052 \mathrm{E}-02$ & $.699661 \mathrm{E}+00$ \\
\hline $.168421 \mathrm{E}-10$ & $.218128 E-04$ & $.347915 \mathrm{E}-02$ & $.349992 \mathrm{E}-02$ & $.699846 \mathrm{E}+00$ \\
\hline $.184211 \mathrm{E}-10$ & $.999453 E-05$ & $.349468 \mathrm{E}-02$ & $.350415 \mathrm{E}-02$ & $.699930 \mathrm{E}+00$ \\
\hline $.200000 \mathrm{E}-10$ & $.459534 \mathrm{E}-05$ & $.350176 \mathrm{E}-02$ & $.350607 \mathrm{E}-02$ & $.699968 \mathrm{E}+00$ \\
\hline$-.100000 \mathrm{E}-10$ & $.186564 E-14$ & $.350162 \mathrm{E}+13$ & $.131230 \mathrm{E}-23$ & $-.842105 E-11$ \\
\hline $.469637 \mathrm{E}-14$ & $.282527 E+13$ & $.162646 \mathrm{E}-23$ & $-.684211 \mathrm{E}-11$ & $.139910 \mathrm{E}-13$ \\
\hline $.178216 E+13$ & $.257843 E-23$ & $-.526316 E-11$ & $.614508 E-13$ & $.681927 E+12$ \\
\hline $.673853 E-23$ & $-.368421 E-11$ & $.628241 \mathrm{E}-12$ & $.103447 E+12$ & $.444204 \mathrm{E}-22$ \\
\hline$-.210526 \mathrm{E}-11$ & $.264816 \mathrm{E}-10$ & $.381484 \mathrm{E}+10$ & $.120456 \mathrm{E}-20$ & $-.526316 \mathrm{E}-12$ \\
\hline $.409317 \mathrm{E}-08$ & $.326591 E+08$ & $.140701 \mathrm{E}-18$ & $.105263 \mathrm{E}-11$ & $.664368 E-06$ \\
\hline $.170982 E+06$ & $.268753 E-16$ & $.263158 \mathrm{E}-11$ & $.332343 E-04$ & $.225786 \mathrm{E}+04$ \\
\hline $.203519 E-14$ & $.421053 E-11$ & $.395905 E-03$ & $.139196 \mathrm{E}+03$ & $.330122 E-13$ \\
\hline $.578947 \mathrm{E}-11$ & $.142363 E-02$ & $.304284 \mathrm{E}+02$ & $.151016 \mathrm{E}-12$ & \\
\hline
\end{tabular}

In a similar manner, we define the following for a reverse biased transistor [10]

$$
\omega_{\alpha \gamma}=\frac{I_{c s}}{\left(S_{n c}+\tilde{S}_{p c}\right) p_{b 0}}
$$


So that

$$
C_{c}=\frac{q I_{c s}}{k T \omega_{\alpha \gamma}} \mathrm{e}^{V_{c b} / V_{T}}
$$

\subsection{Static Model}

The continuity equation and the current density equation govern the steady state characteristics. The terminal currents after solving these equations are given by:

$$
\begin{aligned}
I_{E}= & A_{q} \frac{D_{B} n_{b}}{L_{B}} \operatorname{coth}\left(\frac{W}{L_{B}}\right)\left[\mathrm{e}^{V_{e b} / V_{T}}-1\right] \\
& -\frac{1}{\cos h\left(w / L_{B}\right)}\left[\mathrm{e}^{V_{c b} / V_{T}}-1\right]+A_{q} \frac{D_{E} P_{E}}{L_{E}}\left[\mathrm{e}^{V_{c b} / V_{T}}-1\right] \\
I_{C}= & A_{q} \frac{D_{B} n_{b}}{\sin h\left(\frac{W}{L_{B}}\right)-L_{B}}\left[\mathrm{e}^{V_{e b} / V_{T}}-1\right]-\operatorname{coth}\left(\frac{W}{L_{B}}\right)\left[\mathrm{e}^{V_{c b} / V_{T}}-1\right] \\
& -A_{q} \frac{D_{c} P_{c}}{L_{c}}\left[\mathrm{e}^{V_{c b} / V_{T}}-1\right]
\end{aligned}
$$

\section{DEVELOPMENT OF NUMERICAL ALGORITHM}

We have solved the following differential equations using finite difference method.

$$
\begin{gathered}
\nu_{0}(x)=n_{p 0} \frac{\sin h\left[(w-x) / L_{n}\right]}{\sin h\left(w / L_{n}\right)} \\
\frac{\partial^{2} \nu_{0}}{\partial x^{2}}-\frac{\nu_{0}}{L_{n}^{2}}=0 \\
\frac{\partial^{2} \nu_{1}}{\partial x^{2}}-\frac{\nu_{1}}{L_{n}^{2}}=\frac{\nu_{0}}{D_{n}} \\
\frac{\partial^{2} \nu_{2}}{\partial x^{2}}-\frac{\nu_{2}}{L_{n}^{2}}=\frac{\nu_{1}}{D_{n}} \\
\frac{\partial^{2} \nu_{3}}{\partial x^{2}}-\frac{\nu_{3}}{L_{n}^{2}}=\frac{\nu_{2}}{D_{n}}
\end{gathered}
$$


The boundary conditions for zero order solution are:

$$
\begin{aligned}
& \nu_{0}(0)=n_{p 0} \\
& \nu_{0}(\omega)=0
\end{aligned}
$$

and for higher order solutions are:

$$
\nu_{n}(0)=\nu_{m}(\omega)=0
$$

We have used the following expressions for electron and hole mobility calculations:

$$
\begin{aligned}
& \mu_{n}=\mu_{0}+\frac{\mu_{1}-\mu_{0}}{\left(1+\frac{N(n)}{\mu_{2}}\right) \mu_{3}} \\
& \mu_{p}=\mu_{4}+\mu_{5}-\mu_{4} /\left(\left[1+N(n) / \mu_{6}\right] \mu_{7}\right)
\end{aligned}
$$

To calculate the minority carrier life times, we use the Shockley Read Hall Recombination Mechanism, the electron and hole life times are expressed as:

$$
\tau_{n}=\frac{\tau_{e}}{1+\frac{N(n)}{N S R H}} ; \quad \tau_{p}=\frac{\tau_{n}}{1+\frac{N(n)}{N S R H}}
$$

We have used Finite difference Method to solve the partial difference equations and used Gauss Elimination Method to solve the matrix generated by FDM and for integration we used Simpson' rule.

Program's data and control flow can be described by the following steps:

Step 1 Read the input data from a file.

Step 2 Convert each second order differential equations into a difference equation using finite difference method and get the equation $A x=B$.

Step 3 Solve the equation $A x=B$ using Gauss Elimination Method to get the position function value at different points in the neutral base region.

Step 4 Repeat Step 1 for other higher order differential equations till the last one. 
Step 5 Use plot routine to get plot of the position function in the neutral base region.

Step 6 Use Simpson's integration method to calculate the coefficients $a 0, a 1, a 2, a 3$.

Step 7 Calculate upto third order derivative of the applied input signal.

Step 8 Calculate base current.

Step 9 Use finite difference method to compute different components of the emitter current upto third order effect at $x=0$.

$$
\begin{aligned}
& i_{e 0}(0, t)=-A_{q} D_{n} \nu_{0}^{\prime}(0) \gamma(t) \\
& i_{e 1}(0, t)=-A_{q} D_{n} \nu_{1}^{\prime}(0) \gamma^{\prime}(t) \\
& i_{e 2}(0, t)=-A_{q} D_{n} \nu_{2}^{\prime}(0) \gamma^{\prime \prime}(t) \\
& i_{e 3}(0, t)=-A_{q} D_{n} \nu_{3}^{\prime}(0) \gamma^{\prime \prime \prime}(t)
\end{aligned}
$$

Step 10 Use finite difference method to compute zero and higher order terms of collector current at $x=w$.

$$
\begin{aligned}
& i_{c 0}(w, t)=-A_{q} D_{n} \nu_{0}^{\prime}(w) \gamma(t) \\
& i_{c 1}(w, t)=-A_{q} D_{n} \nu_{1}^{\prime}(w) \gamma^{\prime}(t) \\
& i_{c 2}(w, t)=-A_{q} D_{n} \nu_{2}^{\prime}(w) \gamma^{\prime \prime}(t) \\
& i_{c 3}(w, t)=-A_{q} D_{n} \nu_{3}^{\prime}(w) \gamma^{\prime \prime \prime}(t)
\end{aligned}
$$

Step 11 Use the character-plot routine to get the plot of these terminal current and signal wave form.

\section{RESULTS}

We have carried out simulation on this package at various frequencies. Simulation results for two examples are given in appendix. In the first example the program takes area, width, minority carrier concentration, life time, diffusion constant and diffusion length as input and does small signal sinusoidal analysis at $8 \mathrm{GHz}$. It calculates the gamma function and its derivatives and plot them against time. Next it use the accurate non-linear model given in (9) to calculate base, emitter and 
collector currents and plots them against time. In the second example the program inputs area, width, minority carrier concentration, base doping, emitter doping and collector doping concentration and life time, diffusion constant and diffusion length in the base region. It does transient analysis. It calculates reverse saturation currents in emitter and collector regions, collector capacitances. Current gain, break down voltages for base emitter and base collector regions, 3- $d B$ cut off frequencies, gamma function and their derivatives. It plots them against time. Next it uses the accurate non-linear model and calculatesbase, collector and emitter currents, emitter resistance, emitter capacitance and plots them against time.

\section{CONCLUSION}

We have developed a high speed bipolar transistor device analysis (HIBTRA) package, which is based on the high speed bipolar transistor modeling via non linear circuit elements. It uses analytical model, numerical model, lumped model and static model of bipolar transistor. The results obtained on the basis of higher order circuit elements is a more accurate circuit representation of bipolar transistor at high frequency, for reliable circuit simulation.

It uses less memory (approximately $86 \mathrm{~KB}$ ) and computation is also fast, while other device simulators require approximately $2 \mathrm{MB}$ core memory, so it requires either XENIX or UNIX based systems. Our developed package incorporates the latest high speed bipolar transistors models, and it also does the physical simulation. It also computes the other device parameters starting from the physical parameters of the bipolar transistor. Plotting facility has been also included in the package to get the dynamic simulation characteristics.

\section{References}

[1] Ebers, J. J. and Moll, J. L. (1954). "Large signal behaviour of junction transistor", Proc. IRE, 42, 1761-1772.

[2] Gray, P. R., Devitt, D., Boothrayd, A. R. and Gibbions, J. F. (1964). Physical Electronics and Circuit Models of Transistors, SEEC, 2, Wiley, NewYork.

[3] Gummel, H. K. and Poon, H. C. (1970). 'An integral charge control of bipolar transistor', Bell Syst. Tech. J., 49, 827-852. 
[4] Vlach, J. and Singhal, K. (1983). Computer Methods for circuit Analysis and Diagram, Van Nostrand and Reinhold, NewYork.

[5] Sze, S. M. (1981). Physics of Semi Conductor Devices, 2nd edn., Wiley New York.

[6] Chua, L. O. (1980). 'Device modelling viz basic non linear circuit elements', IEEE Transcircuit and Systems, CAS-27, pp. 1014-1044.

[7] Philippow, E. and Jakubenko, W. (1983). 'Ein Bertrag Zur Verwendung von Elementin Hoherer Ordrung Zur Modellierung des Pipclartransistors', Z. Eklekr, Inform-U Energies Technik, 13, 395-404.

[8] Chua, L. O. and Chang, C. C. (1988). 'High-Speed non linear circuit models for $p-n$ junction diodes', Int. J. ckt. Theory Appl., 16, 157-190.

[9] Sipila, M., Porra, V. and Valtonen, M. (1989). 'Improved Description of Base Dynamics in the modelling of bipolar transistors', International Journal of Circuit Theory and Applications, 17, 465-482.

[10] Device Modelling (chap. V), Computer Aided Network Design Donald A. Calahan, (1st Edition), T.M.H. Edition. 

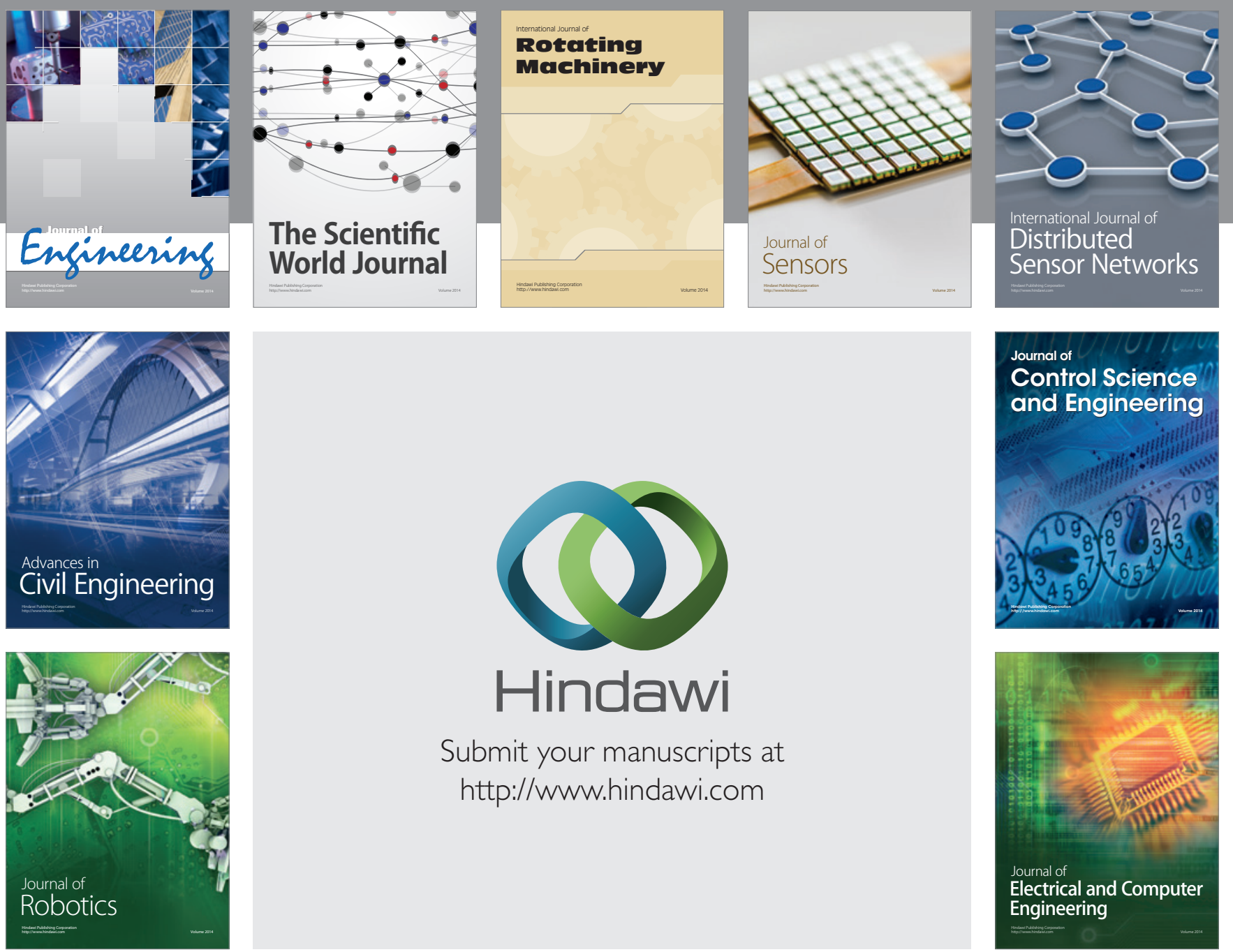

Submit your manuscripts at

http://www.hindawi.com
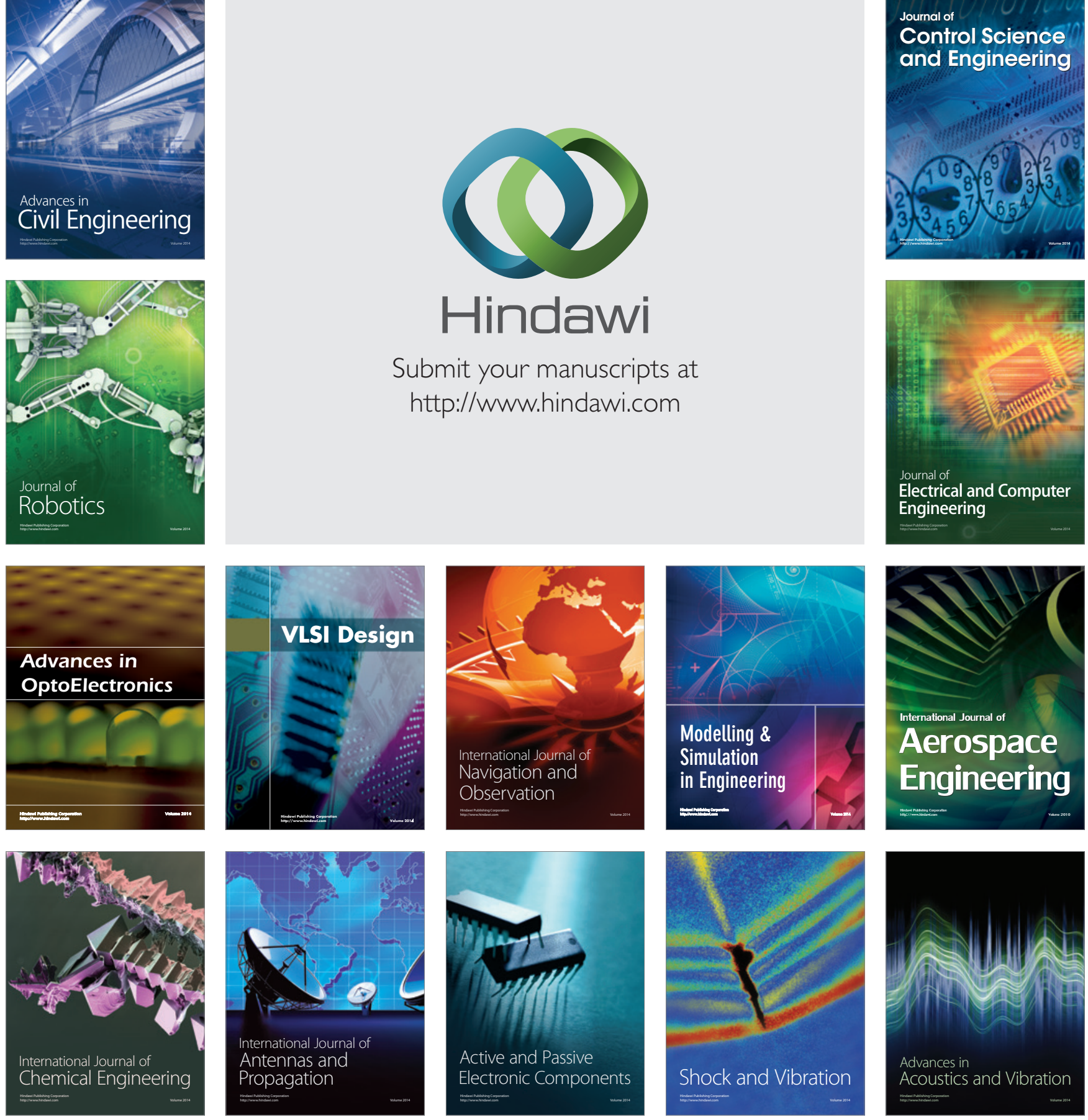